\title{
Cause-and-Effect Relationships in Dry Beans Cultivars Yield Components Under Crop-Livestock System Management
}

\author{
Sarha G. K. Kobata ${ }^{1}$, Betania B. de Bortolli ${ }^{1}$, Heloíze Dums ${ }^{1}$, Marcos A. de Bortolli ${ }^{1}$, Tangriani S. Assmann ${ }^{1}$, \\ Amanda Pavan ${ }^{1} \&$ André B. Soares ${ }^{1}$ \\ ${ }^{1}$ Federal University of Technology, Paraná, Brazil \\ Correspondence: Sarha G. K. Kobata, Federal University of Technology, Paraná, Brazil. Tel: 55-449-9989-6028. \\ E-mail: kobata.sarha@gmail.com
}

Received: December 18, 2018

Accepted: January 20, 2019 Online Published: March 15, 2019

doi:10.5539/jas.v11n4p525

URL: https://doi.org/10.5539/jas.v11n4p525

\begin{abstract}
In order to identify the contribution of yield components to the final yield of dry beans, two widely adopted cultivars (IAC Milênio, and IPR Tuiuiú) were evaluated under nitrogen fertilization levels $(0,50,100$ and $150 \mathrm{~kg}$ $\mathrm{N} \mathrm{ha}^{-1}$ ) in a crop-livestock system. Experiments were conducted under a randomized complete block with three replications design during two years (2016 and 2017) which were split in two cropping phases. A subplot factorial scheme of grazing heights by nitrogen fertilization (grains crops or pasture) was used. Dry beans crops were fertilized during the summer and yield components evaluated along with yield measurements. Descriptive and Pearson's correlation coefficient analyses were performed and followed by path analysis to explain the interrelationship between yield components (explanatory traits) and yield (main trait). For IAC Milenio in 2016 cultivated as a second crop the main trait influencing yield the number of seeds per plant through direct and indirect effect of other traits, regardless of the topdressing nitrogen level applied. In 2017, a greater number of significant correlations was found for the IPR Tuiuiú cultivar. The number of pods per plant was the trait that affected yield most all nitrogen levels.
\end{abstract}

Keywords: crop-livestock system, nitrogen fertilization, path analysis

\section{Introduction}

Brazil produced 1.7 million tons of first crop beans in the 2017/2018 season. Total production derived from two marked harvest seasons: a first one, harvested in January-February that yielded $1207 \mathrm{~kg} \mathrm{ha}^{-1}$ and a second crop yielding $850 \mathrm{~kg} \mathrm{ha}^{-1}$ harvested around March. Paraná state is the major producer of the commodity, producing a sizable crop on both harvests (CONAB, 2018). The national and state average yield is perceived as low, given that $3000 \mathrm{~kg} \mathrm{ha}^{-1}$ is currently attained in farms that adopt high technology agronomic practices (Silva, Lemos, \& Crusciol, 2011).

According to SEAB (2017), the dry bean crop is an attractive economic alternative for small farms that generates significant employment in the state. It is known, though, that its maximum potential yield is substantially higher than observed yields. This yield gap can be lowered through the use of nitrogen fertilizers and enhanced cultivars in low-tech settings (Teixeira, Andrade, Carvalho, Morais, \& Corrêa, 2000).

Fertilization is mandatory for high-yielding beans in crop-livestock systems, but it is also common practice to take advantage of the residual effect of previous cool-season pasture nitrogen fertilization on the following beans crop. Residual effect from fertilization showed beneficial effects on a following oats crop after corn (Adami et al., 2012).

Previous literature support a linear relationship between nitrogen fertilization rates and dry beans yield, while recent studies corroborate this finding adding that the relationship holds true up to $120 \mathrm{~kg} \mathrm{ha}^{-1}$ under no-till (Gomes Junior, De Sá, \& Valério Filho, 2008) and $98 \mathrm{~kg} \mathrm{ha}^{-1}$ in conventional systems (Viana et al., 2011)

Costa and Zimmermann (1988) showed the importance of yield components-grain specific weight, pods per plant, grains per plant — on the bean crop productivity. Based on the correlation between those components and yield, it is possible to identify those traits that should be aimed for in a genetic improvement program (Hoogerheide, Vencovsky, Farias, Freire, \& Arantes, 2007). Through this type of study, plant breeders can identify side-effect characteristics changes that are a consequence of selecting for another trait (Ramalho, Santos, \& Zimmermann, 1993). On top of that, the correlation between the measured traits are the groundwork for building 
statistical protocols for dry beans research, since it points to what traits should be evaluated for a desired phenotype.

The linear correlation coefficient suggests that a linear relation between two traits exists. Assuming that a cause-and-effect relationship is always present can lead to erroneous trait selection, since a third trait or group of traits can be highly correlated with the two traits being analyzed. In order to elucidate the third-party effects on studied traits, "Path Analysis" has been used (Cruz \& Carneiro, 2012).

This technique has been used in various crops recently: dry beans (Cabral, Soares, Lima, Soares, \& Silva, 2011; Kurek, Carvalho, Assmann, Marchioro, \& Cruz, 2001; Zilio, Coelho, Souza, Santos, \& Miquelluti, 2011); soybeans (de Carvalho, Arias, Toledo, Oliveira, \& Vello, 2002; Nogueira et al., 2012), wheat (Gondim, Rocha, Sediyama, \& Miranda, 2008), canola (Coimbra et al., 2005), and corn (Lopes et al., 2007). These studies prove that path analysis can identify the appropriate yield components in supporting a more efficient genetic improvement program.

This study aimed to identify the cause-and-effect relationship between yield components and seed yield per plant of two dry beans cultivars planted after pasture that was managed under two grazing heights and topdressing nitrogen doses as part of a long-term crop-livestock system study.

\section{Materials and Methods}

\subsection{Experimental Site}

The experiment was conducted in Abelardo Luz, Santa Catarina state $\left(26^{\circ} 31^{\prime} \mathrm{S}, 51^{\circ} 35^{\prime} \mathrm{W} ; 850 \mathrm{~m}\right)$. Total experimental area was 20 ha, which has been managed under crop-livestock and no-till planting since 2012. This study collected beans data during two consecutive years, according to the crop-livestock rotation protocol presented on Table 1.

Table 1. Crop and rotation schedule. Location: UTFPR, Pato Branco campus-2018

\begin{tabular}{|c|c|c|}
\hline Season & Cultivation phase & Crop \\
\hline \multirow[t]{3}{*}{$\mathrm{I}$} & Winter-2015 & Ryegrass under grazing \\
\hline & Summer 2015/2016 & Corn for silage \\
\hline & & Beans (IAC Milenio) \\
\hline \multirow[t]{2}{*}{ II } & Winter-2016 & Ryegrass under grazing \\
\hline & Summer 2016/2017 & Beans (IPR Tuiuiú) \\
\hline
\end{tabular}

\subsection{Experimental Design}

A randomized complete block design with three replications was used. In each year (I, II), there were two cultivation phases. In the first phase, during the winter (2015) ryegrass was grown and grazed. Afterward, during the spring/summer (2015/2016), corn for silage followed by dry beans was cropped. During the second year, ryegrass was grown and grazed again (2016), and the subsequent summer crop was early planted dry beans.

Treatments during the grazing pre-experimental phase were the factorial combination of two sward heights (high = $25 \mathrm{~cm}$, low $=15 \mathrm{~cm})$ and two nitrogen fertilization levels $\left(0\right.$ and $\left.200 \mathrm{~kg} \mathrm{~N} \mathrm{~h}^{-1}\right)$. The nitrogen level treatment was applied to the winter pasture using $45 \% \mathrm{~N}$-urea at the tillering stage. During the first year, corn was planted and submitted to nitrogen rates treatments $\left(0\right.$ and $200 \mathrm{~kg} \mathrm{~N}^{-1}$, urea) at V3 stage. However, the $200 \mathrm{~kg}$ dosis was applied to parcels which did not receive nitrogen during the prior grazing period. Conversely, plots that did receive nitrogen during the grazing period were not fertilized when cultivated with corn afterward.

After corn harvest (year I) and grazers removal followed by ryegrass desiccation (year II), dry beans was planted. On the twelve experimental units resultant from the treatment combinations applied to the pasture and silage corn, four nitrogen fertilization levels $(0,50,100$, and 150) were applied to the subplots planted with dry beans.

In 2016 dry beans was planted as a second crop succeeding corn for silage, which received $200 \mathrm{~kg} \mathrm{~N} \mathrm{ha}^{-1}$ at V5 stage through one application. In 2017 dry beans was planted after ryegrass that was fertilized with $200 \mathrm{~kg} \mathrm{~N} \mathrm{ha}^{-1}$.

Data was collected from the dry beans crop, cultivar IAC Milênio (2016) and IPR Tuiuiú (2017) planted on 27/01/2016 (2016) and 01/12/2016 (2017). On both planting occasions no-tillage was used, and row spacing of $0.45 \mathrm{~m}$ adopted. NPK at the 2-20-20 in 2016, and 8-20-20 concentration in 2017 was the side-dressing fertilization scheme adopted. 
At the time of beans harvest, 60 (2016) and 96 (2017) plants were sampled on each nitrogen fertilization level. Data collected for these samples were: plant height $(\mathrm{H})$, first pod height $(\mathrm{FPH})$, pods per plant $(\mathrm{P})$, seed per pod (SP), seeds per plant (S), pod weight per plant (PW), seeds yield per plant (SY) and thousand seeds weight (SW).

\subsection{Data Analysis}

Descriptive statistics were investigated followed by the analysis of the Pearson's linear correlation coefficient between yield components. After obtaining the correlation coefficients and correcting for multicollinearity between the traits, the direct and indirect influencers on SY were established via path analysis.

The condition number (Montgomery \& Peck, 1981) was used to assess the multicollinearity of the linear simple correlation matrix for the traits. Only traits with weak multicollinearity were kept for further analysis. For season I, at nitrogen dosis 0 , no traits were removed. At $50 \mathrm{~kg} \mathrm{~N} \mathrm{ha}^{-1}, \mathrm{~S}$ and $\mathrm{PW}$ were removed. At $100 \mathrm{~kg} \mathrm{~N} \mathrm{ha}^{-1} \mathrm{~S}$ and SW did not fall under "weak" and at $150 \mathrm{~kg} \mathrm{~N} \mathrm{ha}^{-1}$, SP. For season II only S was removed, but for all nitrogen levels. Further path analysis analysis was carried without redundant (those which caused multicollinearity) characters. SY was found to be the main trait, and the explanatory traits were the yield components. All statistical analyses were conducted using the Genes software (Cruz, 2013).

\section{Results and Discussion}

Coefficient of variation for studied traits can be classified as high or moderately high overall (Table 2). In subplots that did not receive nitrogen fertilization in 2016 (season I), FPH had the greatest coefficient of variation (84.94\%). Under $50 \mathrm{~kg} \mathrm{~N} \mathrm{ha}^{-1}$, the greatest variability $(\mathrm{CV}=46.21 \%)$ was found for $\mathrm{P}$, just like in Moreira, Pegoraro, Vieira, Borges, and Kondo (2013). Under $100 \mathrm{~kg} \mathrm{~N} \mathrm{ha}^{-1}, \mathrm{SW}$ was the trait with largest intrinsic variance $(\mathrm{CV}=81.0 \%)$ and SY presented the largest CV (37.9\%) under the higher nitrogen treatment.

Table 2. Descriptive statistics for seeds yield per plant (SY), plant height (H), first pod height (FPH), pods per plant $(\mathrm{P})$, seed per pod (SP), seeds per plant (S), pod weight per plant (PW) and thousand seeds weight (SW) for dry beans (Year I, $\mathrm{n}=60$ plants, cultivar IAC Milênio; Year II, $\mathrm{n}=96$ plants, Cultivar IPR Tuiuiú) under four nitrogen dosages $\left(0,50,100\right.$ and $\left.150 \mathrm{~kg} \mathrm{ha}^{-1}\right)$. Abelardo Luz-SC, 2016/2017

\begin{tabular}{|c|c|c|c|c|c|c|c|c|c|c|c|c|}
\hline \multirow{3}{*}{ Character } & \multicolumn{12}{|c|}{ Dosages } \\
\hline & \multicolumn{3}{|c|}{0} & \multicolumn{3}{|c|}{50} & \multicolumn{3}{|c|}{100} & \multicolumn{3}{|c|}{150} \\
\hline & Mean & $\mathrm{CV}$ & SD & Mean & $\mathrm{CV}$ & SD & Mean & $\mathrm{CV}$ & SD & Mean & $\mathrm{CV}$ & SD \\
\hline \multicolumn{13}{|c|}{ Year I-Cultivar IAC Milênio } \\
\hline SY & 10.54 & 50.18 & 5.29 & 12.68 & 39.72 & 5.04 & 14.56 & 46.08 & 6.71 & 13.94 & 37.92 & 5.29 \\
\hline $\mathrm{H}$ & 64.47 & 21.75 & 14.02 & 72.15 & 16.97 & 12.25 & 75.48 & 25.41 & 19.18 & 70.30 & 18.55 & 13.04 \\
\hline FPH & 11.12 & 84.92 & 9.44 & 8.80 & 40.56 & 3.57 & 8.30 & 42.22 & 3.50 & 7.67 & 30.35 & 2.33 \\
\hline $\mathrm{P}$ & 10.02 & 47.35 & 4.74 & 12.27 & 46.21 & 5.67 & 13.77 & 42.55 & 5.86 & 13.47 & 35.98 & 4.84 \\
\hline SP & 4.17 & 25.20 & 1.05 & 3.99 & 19.94 & 0.80 & 4.06 & 16.88 & 0.69 & 3.97 & 20.49 & 0.81 \\
\hline $\mathrm{S}$ & 41.70 & 51.65 & 21.54 & 48.50 & 45.04 & 21.85 & 55.85 & 46.18 & 25.79 & 52.88 & 37.19 & 19.67 \\
\hline PW & 1.11 & 33.58 & 0.37 & 1.09 & 32.52 & 0.35 & 1.14 & 70.85 & 0.81 & 1.04 & 20.45 & 0.21 \\
\hline SW & 258.59 & 16.39 & 42.38 & 273.45 & 28.68 & 78.41 & 288.18 & 81.03 & 233.51 & 266.27 & 16.08 & 42.81 \\
\hline \multicolumn{13}{|c|}{ Year II-Cultivar IPR Tuiuiú } \\
\hline SY & 9.94 & 52.39 & 5.21 & 14.96 & 58.72 & 8.78 & 14.94 & 58.05 & 8.68 & 14.94 & 57.11 & 8.53 \\
\hline $\mathrm{H}$ & 52.66 & 19.42 & 10.22 & 58.59 & 20.94 & 12.27 & 60.51 & 18.07 & 10.93 & 58.75 & 19.74 & 11.6 \\
\hline FPH & 19.8 & 18.58 & 3.68 & 19.99 & 21.78 & 4.35 & 19.01 & 22.34 & 4.25 & 19.75 & 22.34 & 4.41 \\
\hline $\mathrm{P}$ & 9.31 & 47.8 & 4.45 & 12.69 & 48.94 & 6.21 & 12.29 & 51.68 & 6.35 & 13.78 & 85.44 & 11.78 \\
\hline SP & 4.86 & 20.15 & 0.98 & 4.91 & 15.14 & 0.74 & 5.14 & 13.14 & 0.68 & 4.71 & 14.9 & 0.7 \\
\hline $\mathrm{S}$ & 44.69 & 50.81 & 22.71 & 63.35 & 55.08 & 34.9 & 63.26 & 54.51 & 34.48 & 66.77 & 90.65 & 60.53 \\
\hline PW & 4.02 & 102.65 & 4.12 & 4.99 & 72.23 & 3.61 & 4.78 & 77.75 & 3.72 & 4.5 & 70.13 & 3.16 \\
\hline SW & 226.55 & 20.58 & 46.62 & 236.58 & 19.87 & 47.00 & 237.47 & 18.14 & 43.07 & 240.73 & 23.77 & 57.21 \\
\hline
\end{tabular}

Note. $\mathrm{CV}=$ coefficient of variation; $\mathrm{SD}=$ standard desviation.

In 2017 (year II), the cultivar IPR Tuiuiú showed the largest coefficient of variation in the subplots without nitrogen application (dosis 0 ) and under the 50 and $100 \mathrm{~kg} \mathrm{ha}^{-1}$ for PW, these being $102.65 \%, 72.23 \%$ and $77.75 \%$ respectively. Under $150 \mathrm{~kg} \mathrm{~N} \mathrm{ha}^{-1}$, S showed the largest coefficient of variation, $90.65 \%$. The second largest $\mathrm{CV}$ in 
the second year was SY under the 0, 50 and $100 \mathrm{~kg} \mathrm{~N} \mathrm{ha}^{-1}$ treatments, these being $52.39 \%, 58.72$ and $58.05 \%$, respectively.

Mean increment on SY was observed when applied nitrogen did not exceed $100 \mathrm{~kg} \mathrm{ha}^{-1}$. For the IPR Tuiuiú cultivar increasing nitrogen past this level had no impact on yield, and for IAC Milenio a reduction in yield was observed. Similar results were found in a study conducted by Gomes Junior et al. (2008), which found a linear relationship between yield and nitrogen level up to $120 \mathrm{~kg} \mathrm{~N} \mathrm{ha}^{-1}$ for two dry beans cultivars under no-till planting and corn stover studying two dry beans cultivars. Viana et al. (2011), which also explored the relationship between $\mathrm{N}$ and dry beans yield (carioca cultivar), found the largest yield under a $98 \mathrm{~kg} \mathrm{~N} \mathrm{ha}^{-1}$ fertilization scheme in an area that was tilled before planting. According to Teixeira, Andrade, Carvalho, Morais, and Corrêa (2000), three main traits explain the increase in dry beans yields: number of pods per plant, number of grains per pod, and 100 grains weight.

$\mathrm{H}$ increased with nitrogen application up to $100 \mathrm{~kg} \mathrm{~N} \mathrm{ha}^{-1}$ for both cultivars, but IAC Milenio presented higher mean values (Table 1).

With increased nitrogen levels reduced FPH was observed for IAC Milenio. IPR Tuiuiú, on the other hand, did not see FPH altered because of nitrogen application but was measured higher consistently across treatment levels. It is known that this trait can be affected by environmental conditions and management during planting (Kappes, Wruck, Carvalho, \& Yamashita, 2008). IPR Tuiuiú was measured for FPH from $13.9 \mathrm{~cm}$ (0 nitrogen) to $24.5 \mathrm{~cm}$ $\left(200 \mathrm{~kg} \mathrm{~N} \mathrm{ha}^{-1}\right.$ ) according to Demari, Souza, Carvalho, Nardino, and Follmann (2015). Plants that have a more upright growth and yield more present greater FPH and should be easier to manage under mechanized harvest, on top of being physiologically more efficient (Moura et al., 2013). IPR Tuiuiú did not respond to nitrogen levels for for FPH, but for IAC Milenio higher FPH was observed when nitrogen was not applied.

For P, both cultivars presented similar mean maxima values: 13.47 (IPR Tuiuiú) and 13.78 (IAC Milenio) under $150 \mathrm{~kg} \mathrm{ha}^{-1}$. Arf et al. (2012) and Teixeira et al. (2000) observed similar results at $80 \mathrm{~kg} \mathrm{ha}^{-1}$ and $150 \mathrm{~kg} \mathrm{ha}^{-1}$ respectively for the Perola cultivar. Similarly, Viana et al. (2011) found 14 pods per plant using $108 \mathrm{~kg} \mathrm{~N} \mathrm{ha}^{-1}$ for the Carioca cultivar.

The trait SP showed a somewhat stable response to the nitrogen treatment. Gomes Junior et al. (2008) did not find significant difference among nitrogen fertilization levels for SP, suggesting that this is a trait highly dependent on the genotype (Arf et al., 2011; Afonso et al., 2011) so its response to environmental changes are mild or non-existent (Souza, Andrade, Vieira, \& Albuquerque, 2008). A similar response was observed for PW, which attained the greatest value at $50 \mathrm{~kg} \mathrm{~N} \mathrm{ha}^{-1}$ for the IPR Tuiuiú cultivar.

The $\mathrm{S}$ trait was measured higher for the IPR Tuiuiú cultivar under the $150 \mathrm{~kg} \mathrm{~N} \mathrm{ha}^{-1}$ level. On the other hand, for the IAC Milenio, the $\mathrm{S}$ trait showed greatest values at $100 \mathrm{~kg} \mathrm{~N} \mathrm{ha}^{-1}$ (56 grains per plant) and was measured lower under $150 \mathrm{~kg} \mathrm{~N} \mathrm{ha}^{-1}$. This is due to the higher nitrogen availability at the early crop stages for the bean crop coming from the previous crop (ryegrass) residue (Afonso et al., 2011).

IAC Milenio achieved higher values for SW (288.2 g) under the $100 \mathrm{~kg} \mathrm{~N} \mathrm{ha}^{-1}$ level. The IPR Tuiuiú cultivar showed a stable trend for this trait since it was planted after ryegrass and the residual effect on the crop nutrition was present throughout all crop stages. Viana et al. (2011) applying a higher $\mathrm{N}$ dose, $140 \mathrm{~kg} \mathrm{~N}$ ha $^{-1}$, weighed $202 \mathrm{~g}$ for 100 seeds.

Pearson product-moment correlation coefficient for the eight measured traits varied from zero (absence of relationship) to 0.98 (strong relationship) (Table 3) for IAC Milenio during year 1 (2016). Under all nitrogen levels, for both cultivars and years, the $\mathrm{P}$ and $\mathrm{S}$ traits were highly correlated with SY supporting similar findings by Cabral et al. (2011). H presented a positive and significant correlation with SY only under the zero nitrogen level, showing that higher plants yield more when nitrogen is absent also yield more. Under the other nitrogen levels there was no significant correlation between $\mathrm{H}$ and SY, meaning that $\mathrm{H}$ does not affect SY when topdressing nitrogen is applied. A marked response of SW to S and SP was observed under all nitrogen levels. As expected, the relationship is inversely proportional which is explained by the source-sink physiology: assimilates are distributed for a greater number of entities and therefore won't fill as much, resulting also in a lower SW. Similar results were observed by Duarte, Peil, and Montezano (2008) for melons, and Zilio et al. (2011) which found SP to influence SW negatively. On the other hand, PW generally increases with SW (Table 3). 
Table 3. Pearson correlation coefficient for yield components (plant height $(\mathrm{H})$, first pod height (FPH), pods per plant (P), seed per pod (SP), seeds per plant (S), pod weight per plant (PW), and thousand seeds weight (SW)) and seed yield per plant (SY) for dry beans, IAC Milenio cultivar (Year 1, $\mathrm{n}=60$ plants) under four nitrogen levels $(0$, 50,100 and $150 \mathrm{~kg} \mathrm{ha}^{-1}$ ). Abelardo Luz-SC, 2016

\begin{tabular}{|c|c|c|c|c|c|c|c|c|}
\hline \multicolumn{9}{|l|}{ Year I } \\
\hline Character & SY & $\mathrm{H}$ & FPH & $\mathrm{P}$ & SP & $\mathrm{S}$ & PW & SW \\
\hline \multicolumn{9}{|c|}{ Dosis $0 \mathrm{~kg} \mathrm{ha}^{-1}$} \\
\hline SY & 1.000 & & & & & & & \\
\hline $\mathrm{H}$ & $0.550^{* *}$ & 1.000 & & & & & & \\
\hline FPH & $-0.157^{\text {ns }}$ & $0.007^{\mathrm{ns}}$ & 1.000 & & & & & \\
\hline $\mathrm{P}$ & $0.827^{* *}$ & $0.551^{* *}$ & $-0.177^{\mathrm{ns}}$ & 1.000 & & & & \\
\hline SP & $0.249^{\text {ns }}$ & $0.259^{*}$ & $0.003^{\mathrm{ns}}$ & $-0.083^{\text {ns }}$ & 1.000 & & & \\
\hline $\mathrm{S}$ & $0.929^{* *}$ & $0.594^{* *}$ & $-0.178^{\mathrm{ns}}$ & $0.848^{* *}$ & $0.330^{* *}$ & 1.000 & & \\
\hline PW & $0.098^{\mathrm{ns}}$ & $-0.169^{\text {ns }}$ & $0.114^{\mathrm{ns}}$ & $-0.354^{* *}$ & $0.250^{\mathrm{ns}}$ & $-0.021^{\mathrm{ns}}$ & 1.000 & \\
\hline SW & $0.064^{\mathrm{ns}}$ & $-0.178^{\text {ns }}$ & $0.069^{\mathrm{ns}}$ & $-0.176^{\text {ns }}$ & $-0.259^{*}$ & $-0.267^{*}$ & $0.400^{* *}$ & 1.000 \\
\hline \multicolumn{9}{|c|}{ Dosis $50 \mathrm{~kg} \mathrm{ha}$} \\
\hline SY & 1.000 & & & & & & & \\
\hline $\mathrm{H}$ & $0.225^{\mathrm{ns}}$ & 1.000 & & & & & & \\
\hline FPH & $-0.077^{\mathrm{ns}}$ & $0.000^{\mathrm{ns}}$ & 1.000 & & & & & \\
\hline $\mathrm{P}$ & $0.567^{* *}$ & $0.251^{\mathrm{ns}}$ & $-0.224^{\mathrm{ns}}$ & 1.000 & & & & \\
\hline SP & $0.345^{* *}$ & $0.238^{\mathrm{ns}}$ & $-0.039^{\mathrm{ns}}$ & $-0.107^{\text {ns }}$ & 1.000 & & & \\
\hline $\mathrm{S}$ & $0.754^{* *}$ & $0.314^{*}$ & $-0.245^{\mathrm{ns}}$ & $0.879^{* *}$ & $0.337^{* *}$ & 1.000 & & \\
\hline PW & $0.387^{* *}$ & $0.024^{\mathrm{ns}}$ & $0.410^{* *}$ & $-0.319^{*}$ & $0.508^{* *}$ & $-0.082^{\mathrm{ns}}$ & 1.000 & \\
\hline SW & $0.227^{\mathrm{ns}}$ & $-0.163^{\mathrm{ns}}$ & $0.453^{* *}$ & $-0.317^{*}$ & $-0.108^{\text {ns }}$ & $-0.343^{* *}$ & $0.791^{* *}$ & 1.000 \\
\hline \multicolumn{9}{|c|}{ Dosis $100 \mathrm{~kg} \mathrm{ha}$} \\
\hline SY & 1.000 & & & & & & & \\
\hline $\mathrm{H}$ & $0.028^{\mathrm{ns}}$ & 1.000 & & & & & & \\
\hline FPH & $-0.176^{\mathrm{ns}}$ & $0.209^{\mathrm{ns}}$ & 1.000 & & & & & \\
\hline $\mathrm{P}$ & $0.778^{* *}$ & $0.096^{\mathrm{ns}}$ & $-0.120^{\mathrm{ns}}$ & 1.000 & & & & \\
\hline SP & $0.162^{\mathrm{ns}}$ & $0.110^{\mathrm{ns}}$ & $-0.057^{\mathrm{ns}}$ & $0.003^{\text {ns }}$ & 1.000 & & & \\
\hline $\mathrm{S}$ & $0.807^{* *}$ & $0.126^{\mathrm{ns}}$ & $-0.151^{\mathrm{ns}}$ & $0.911^{* *}$ & $0.379^{* *}$ & 1.000 & & \\
\hline PW & $0.329^{*}$ & $-0.111^{\text {ns }}$ & $-0.066^{\mathrm{ns}}$ & $-0.247^{\text {ns }}$ & $0.017^{\text {ns }}$ & $-0.192^{\mathrm{ns}}$ & 1.000 & \\
\hline SW & $0.287^{*}$ & $-0.144^{\mathrm{ns}}$ & $-0.058^{\mathrm{ns}}$ & $-0.240^{\mathrm{ns}}$ & $-0.176^{\mathrm{ns}}$ & $-0.259^{*}$ & $0.980^{* *}$ & 1.000 \\
\hline \multicolumn{9}{|c|}{ Dosis $150 \mathrm{~kg} \mathrm{ha}$} \\
\hline SY & 1.000 & & & & & & & \\
\hline $\mathrm{H}$ & $0.119^{\text {ns }}$ & 1.000 & & & & & & \\
\hline FPH & $-0.040^{\mathrm{ns}}$ & $0.377^{* *}$ & 1.000 & & & & & \\
\hline $\mathrm{P}$ & $0.807^{* *}$ & $0.161^{\text {ns }}$ & $-0.004^{\mathrm{ns}}$ & 1.000 & & & & \\
\hline SP & $0.248^{\text {ns }}$ & $0.065^{\mathrm{ns}}$ & $-0.040^{\mathrm{ns}}$ & $-0.171^{\text {ns }}$ & 1.000 & & & \\
\hline $\mathrm{S}$ & $0.920^{* *}$ & $0.196^{\mathrm{ns}}$ & $0.015^{\mathrm{ns}}$ & $0.797^{* *}$ & $0.402^{* *}$ & 1.000 & & \\
\hline PW & $0.455^{* *}$ & $0.006^{\mathrm{ns}}$ & $-0.092^{\mathrm{ns}}$ & $-0.120^{\mathrm{ns}}$ & $0.676^{* *}$ & $0.308^{*}$ & 1.000 & \\
\hline SW & $0.206^{\mathrm{ns}}$ & $-0.101^{\mathrm{ns}}$ & $-0.123^{\mathrm{ns}}$ & $0.042^{\text {ns }}$ & $-0.427^{* *}$ & $-0.168^{\mathrm{ns}}$ & $0.348^{* *}$ & 1.000 \\
\hline
\end{tabular}

For year II (2017) (Table 4) and cultivar IPR Tuiuiú, it can be said that SY increased as a consequence of changes in P, S, PW and SW, like observed by Cabral et al. (2011) evaluating 58 genotypes of dry beans under nitrogen dosages (50,100 and $\left.150 \mathrm{~kg} \mathrm{~N} \mathrm{ha}^{-1}\right)$.

A very strong (Carvalho, Lorencetti, \& Benin, 2004) linear correlation $(>0.9)$ was found between $\mathrm{P}$ and $\mathrm{S}$ under all nitrogen levels. The same association level $(\mathrm{r} \geq 0.9)$ was also observed between $\mathrm{S}$ and $\mathrm{SW}$ under 0,50 , and $100 \mathrm{~kg}$ $\mathrm{N} \mathrm{ha}^{-1}$. Yet, this association level decreases $(\mathrm{r}=0.52)$ when the nitrogen level increased to $150 \mathrm{~kg} \mathrm{~N} \mathrm{ha}^{-1}$ (Table 4). This is probably due to assimilates translocation from grain formation to grain filling.

Correlation coefficients shed light on linear relationships between traits but they do not support conclusions about cause-effect (Cabral et al., 2011). Path analysis was therefore conducted to find the cause and effect mapping of the studied variables. 
Table 4. Pearson correlation coefficient for yield components (plant height $(\mathrm{H})$, first pod height (FPH), pods per plant (P), seed per pod (SP), seeds per plant (S), pod weight per plant (PW), and thousand seeds weight (SW)) and seed yield per plant (SY) for dry beans, IAC Milenio cultivar (Year 2, $\mathrm{n}=96$ plants) under four nitrogen levels ( 0 , 50, 100 and $150 \mathrm{~kg} \mathrm{ha}^{-1}$ ). Abelardo Luz-SC, 2017

\begin{tabular}{|c|c|c|c|c|c|c|c|c|}
\hline \multicolumn{9}{|l|}{ Year II } \\
\hline Character & SY & $\mathrm{H}$ & FPH & $\mathrm{P}$ & $\mathrm{SP}$ & $\mathrm{S}$ & $\mathrm{PW}$ & SW \\
\hline \multicolumn{9}{|c|}{ Dosis $0 \mathrm{~kg} \mathrm{ha}^{-1}$} \\
\hline SY & 1.000 & & & & & & & \\
\hline $\mathrm{H}$ & $0.250^{*}$ & 1.000 & & & & & & \\
\hline FPH & $-0.105^{\mathrm{ns}}$ & $-0.042^{\mathrm{ns}}$ & 1.000 & & & & & \\
\hline $\mathrm{P}$ & $0.869^{* *}$ & $0.278^{* *}$ & $-0.122^{\mathrm{ns}}$ & 1.000 & & & & \\
\hline SP & $0.112^{\text {ns }}$ & $0.110^{\text {ns }}$ & $-0.069^{\mathrm{ns}}$ & $-0.153^{\mathrm{ns}}$ & 1.000 & & & \\
\hline S & $0.914^{* *}$ & $0.287^{* *}$ & $-0.129^{\mathrm{ns}}$ & $0.904^{* *}$ & $0.240^{*}$ & 1.000 & & \\
\hline PW & $0.515^{* *}$ & $0.228^{*}$ & $-0.194^{\mathrm{ns}}$ & $0.650^{* *}$ & $-0.156^{\mathrm{ns}}$ & $0.537^{* *}$ & 1.000 & \\
\hline SW & $0.205^{*}$ & $-0.079^{\mathrm{ns}}$ & $0.070^{\mathrm{ns}}$ & $-0.064^{\mathrm{ns}}$ & $-0.360^{* *}$ & $-0.180^{\mathrm{ns}}$ & $-0.035^{\mathrm{ns}}$ & 1.000 \\
\hline \multicolumn{9}{|c|}{ Dosis $50 \mathrm{~kg} \mathrm{ha}^{-1}$} \\
\hline SY & 1.000 & & & & & & & \\
\hline $\mathrm{H}$ & $0.375^{* *}$ & 1.000 & & & & & & \\
\hline FPH & $-0.015^{\mathrm{ns}}$ & $0.209^{*}$ & 1.000 & & & & & \\
\hline $\mathrm{P}$ & $0.929^{* *}$ & $0.409^{* *}$ & $-0.020^{\mathrm{ns}}$ & 1.000 & & & & \\
\hline SP & $0.350^{* *}$ & $0.237^{*}$ & $0.109^{\mathrm{ns}}$ & $0.228^{*}$ & 1.000 & & & \\
\hline S & $0.932^{* *}$ & $0.413^{* *}$ & $0.013^{\mathrm{ns}}$ & $0.954^{* *}$ & $0.471^{* *}$ & 1.000 & & \\
\hline PW & $0.701^{* *}$ & $0.346^{* *}$ & $-0.020^{\mathrm{ns}}$ & $0.647^{* *}$ & $0.085^{\mathrm{ns}}$ & $0.598^{* *}$ & 1.000 & \\
\hline SW & $0.311^{* *}$ & $-0.114^{\mathrm{ns}}$ & $-0.139^{\mathrm{ns}}$ & $0.062^{\mathrm{ns}}$ & $-0.227^{*}$ & $-0.020^{\mathrm{ns}}$ & $0.270^{* *}$ & 1.000 \\
\hline \multicolumn{9}{|c|}{ Dosis $100 \mathrm{~kg} \mathrm{ha}$} \\
\hline SY & 1.000 & & & & & & & \\
\hline $\mathrm{H}$ & $0.162^{\mathrm{ns}}$ & 1.000 & & & & & & \\
\hline FPH & $-0.145^{\mathrm{ns}}$ & $0.148^{\mathrm{ns}}$ & 1.000 & & & & & \\
\hline $\mathrm{P}$ & $0.907^{* *}$ & $0.190^{\mathrm{ns}}$ & $-0.226^{*}$ & 1.000 & & & & \\
\hline SP & $0.207^{*}$ & $0.046^{\mathrm{ns}}$ & $0.152^{\mathrm{ns}}$ & $0.008^{\mathrm{ns}}$ & 1.000 & & & \\
\hline $\mathrm{S}$ & $0.941^{* *}$ & $0.178^{\mathrm{ns}}$ & $-0.183^{\mathrm{ns}}$ & $0.967^{* *}$ & $0.228^{*}$ & 1.000 & & \\
\hline PW & $0.888^{* *}$ & $0.186^{\mathrm{ns}}$ & $-0.186^{\mathrm{ns}}$ & $0.851^{* *}$ & $0.038^{\mathrm{ns}}$ & $0.839^{* *}$ & 1.000 & \\
\hline SW & $0.258^{*}$ & $-0.026^{\mathrm{ns}}$ & $0.036^{\mathrm{ns}}$ & $-0.053^{\mathrm{ns}}$ & $-0.071^{\mathrm{ns}}$ & $-0.053^{\mathrm{ns}}$ & $0.188^{\mathrm{ns}}$ & 1.000 \\
\hline \multicolumn{9}{|c|}{ Dosis $150 \mathrm{~kg} \mathrm{ha}$} \\
\hline SY & 1.000 & & & & & & & \\
\hline $\mathrm{H}$ & $0.328^{* *}$ & 1.000 & & & & & & \\
\hline FPH & $-0.007^{\mathrm{ns}}$ & $0.126^{\mathrm{ns}}$ & 1.000 & & & & & \\
\hline $\mathrm{P}$ & $0.499^{* *}$ & $0.116^{\mathrm{ns}}$ & $-0.019^{\mathrm{ns}}$ & 1.000 & & & & \\
\hline SP & $0.424^{* *}$ & $0.346^{* *}$ & $0.089^{\mathrm{ns}}$ & $0.221^{*}$ & 1.000 & & & \\
\hline S & $0.523^{* *}$ & $0.141^{\mathrm{ns}}$ & $-0.009^{\mathrm{ns}}$ & $0.990^{* *}$ & $0.340^{* *}$ & 1.000 & & \\
\hline PW & $0.893^{* *}$ & $0.294^{* *}$ & $-0.009^{\mathrm{ns}}$ & $0.435^{* *}$ & $0.306^{* *}$ & $0.450^{* *}$ & 1.000 & \\
\hline SW & $0.277^{* *}$ & $0.107^{\mathrm{ns}}$ & $0.079^{\mathrm{ns}}$ & $-0.302^{* *}$ & $-0.169^{\mathrm{ns}}$ & $-0.331^{* *}$ & $0.239^{*}$ & 1.000 \\
\hline
\end{tabular}

In both years, traits can explain 75 to $98 \%$ of the SY variation, depending on the nitrogen level used.

In the first year (2016), for IAC Milenio under zero nitrogen it was observed that positive and significant correlations between $\mathrm{H}$ and $\mathrm{P}$ with SY were mediated by an indirect effect of S (Table 5). In other words, higher plants present greater pods and seeds per plant resulting in higher yield.

Under $50 \mathrm{~kg} \mathrm{~N} \mathrm{ha}^{-1} \mathrm{P}$ and SP were significantly associated with SY. For both, the main reason for the relationship was the direct effect on SY. As pointed by Vale et al. (2009), when the correlation coefficient between a trait and the main trait is similar or equal to its direct effect in both direction and magnitude, the correlation explains the relationship. 
Table 5. Pearson correlation coefficient estimates and associated indirect and direct effects of plant height $(\mathrm{H})$, first pod height (FPH), pods per plant (P), seed per pod (SP), seeds per plant (S), pod weight per plant (PW), and thousand seeds weight (SW) on seed yield per plant (SY) for dry beans, IAC Milenio cultivar (Year 1, $\mathrm{n}=60$ plants) under four nitrogen levels $\left(0,50,100\right.$ and $\left.150 \mathrm{~kg} \mathrm{ha}^{-1}\right)$. Abelardo Luz-SC, 2016

\begin{tabular}{|c|c|c|c|c|c|c|c|}
\hline \multicolumn{8}{|l|}{ Year I } \\
\hline \multirow{2}{*}{ Effect } & \multicolumn{7}{|c|}{ Yield Components } \\
\hline & $\mathrm{H}$ & FPH & $\mathrm{P}$ & SP & $\mathrm{S}$ & PW & SW \\
\hline \multicolumn{8}{|l|}{ Dosis $0 \mathrm{~kg} \mathrm{ha}^{-1}$} \\
\hline DIRECT ON SY & -0.007 & -0.003 & 0.288 & 0.094 & 0.740 & 0.067 & 0.308 \\
\hline INDIRECT VIA H & - & 0.000 & -0.004 & -0.002 & -0.004 & 0.001 & 0.001 \\
\hline INDIRECT VIA FPH & 0.000 & - & 0.001 & 0.000 & 0.001 & 0.000 & 0.000 \\
\hline INDIRECT VIA P & 0.159 & -0.051 & - & -0.024 & 0.244 & -0.102 & -0.051 \\
\hline INDIRECT VIA SP & 0.024 & 0.000 & -0.008 & - & 0.031 & 0.023 & -0.024 \\
\hline INDIRECT VIA S & 0.440 & -0.132 & 0.628 & 0.245 & - & -0.015 & -0.198 \\
\hline INDIRECT VIA PW & -0.011 & 0.008 & -0.024 & 0.017 & -0.001 & - & 0.027 \\
\hline INDIRECT VIA SW & -0.055 & 0.021 & -0.054 & -0.080 & -0.082 & 0.123 & - \\
\hline $\mathrm{r}$ & $0.550^{* *}$ & $-0.157^{\mathrm{ns}}$ & $0.827 * *$ & $0.249^{\mathrm{ns}}$ & $0.929^{* *}$ & $0.098^{\mathrm{ns}}$ & $0.064^{\mathrm{ns}}$ \\
\hline $\mathrm{R}^{2}$ & 0.972 & & & & & & \\
\hline \multicolumn{8}{|l|}{ Dosis $50 \mathrm{~kg} \mathrm{ha}^{-1}$} \\
\hline DIRECT ON SY & 0.014 & -0.156 & 0.769 & 0.483 & - & - & 0.596 \\
\hline INDIRECT VIA H & - & 0.000 & 0.004 & 0.003 & - & - & -0.002 \\
\hline INDIRECT VIA FPH & 0.000 & - & 0.035 & 0.006 & - & - & -0.071 \\
\hline INDIRECT VIA P & 0.193 & -0.172 & - & -0.083 & - & - & -0.244 \\
\hline INDIRECT VIA SP & 0.115 & -0.019 & -0.052 & - & - & - & -0.052 \\
\hline INDIRECT VIA S & - & - & - & - & - & - & - \\
\hline INDIRECT VIA PW & - & - & - & - & - & - & - \\
\hline INDIRECT VIA SW & -0.097 & 0.270 & -0.189 & -0.064 & - & - & - \\
\hline $\mathrm{r}$ & $0.225^{\mathrm{ns}}$ & $-0.077^{\mathrm{ns}}$ & $0.567 * *$ & $0.345 * *$ & - & - & $0.227^{\mathrm{ns}}$ \\
\hline $\mathrm{R}^{2}$ & 0.753 & & & & & & \\
\hline \multicolumn{8}{|l|}{ Dosis $100 \mathrm{~kg} \mathrm{ha}^{-1}$} \\
\hline DIRECT ON SY & -0.011 & -0.019 & 0.912 & 0.150 & - & 0.549 & - \\
\hline INDIRECT VIA H & - & -0.002 & -0.001 & -0.001 & - & 0.001 & - \\
\hline INDIRECT VIA FPH & -0.004 & - & 0.002 & 0.001 & - & 0.001 & - \\
\hline INDIRECT VIA P & 0.088 & -0.110 & - & 0.002 & - & -0.225 & - \\
\hline INDIRECT VIA SP & 0.017 & -0.009 & 0.000 & - & - & 0.003 & - \\
\hline INDIRECT VIA S & - & - & - & - & & - & - \\
\hline INDIRECT VIA PW & -0.061 & -0.036 & -0.136 & 0.009 & & - & - \\
\hline INDIRECT VIA SW & - & - & - & - & & - & - \\
\hline r & $0.028^{\mathrm{ns}}$ & $-0.176^{\mathrm{ns}}$ & $0.778 * *$ & $0.162^{\mathrm{ns}}$ & - & $0.329 *$ & - \\
\hline $\mathrm{R}^{2}$ & 0.918 & & & & & & \\
\hline \multicolumn{8}{|l|}{ Dosis $150 \mathrm{~kg} \mathrm{ha}^{-1}$} \\
\hline DIRECT ON SY & -0.042 & 0.014 & 0.298 & - & 0.665 & 0.206 & 0.232 \\
\hline INDIRECT VIA H & - & -0.016 & -0.007 & - & -0.008 & 0.000 & 0.004 \\
\hline INDIRECT VIA FPH & 0.005 & - & 0.000 & - & 0.000 & -0.001 & -0.002 \\
\hline INDIRECT VIA P & 0.048 & -0.001 & - & - & 0.237 & -0.035 & 0.012 \\
\hline INDIRECT VIA SP & - & - & - & - & - & - & - \\
\hline INDIRECT VIA S & 0.130 & 0.010 & 0.531 & - & - & 0.206 & -0.112 \\
\hline INDIRECT VIA PW & 0.001 & -0.018 & -0.025 & - & 0.064 & - & 0.071 \\
\hline INDIRECT VIA SW & -0.024 & -0.028 & 0.010 & - & -0.039 & 0.080 & - \\
\hline $\mathrm{r}$ & $0.119^{\mathrm{ns}}$ & $-0.040^{\mathrm{ns}}$ & $0.807^{* *}$ & - & $0.920^{* *}$ & $0.455^{* *}$ & $0.206^{\mathrm{ns}}$ \\
\hline $\mathrm{R}^{2}$ & 0.988 & & & & & & \\
\hline
\end{tabular}

When the treatment dosage increased to $100 \mathrm{~kg} \mathrm{~N} \mathrm{ha}^{-1}$, the largest significant positive correlation found $(\mathrm{r}=0.778)$ was of P on SY. The direct effect of P on SY (0.912) was the major cause of the correlation. It can be noticed that PW had a negative impact (-0.136) on SY, via indirect effect on P, reducing the overall correlation, scenario also 
found by Zilio et al. (2011). It is safe to argue that the major mechanism behind these findings is the compensation P vs. SP and SW, already discussed above and explained in great detail by Freitas et al. (2016). PW also showed positive and significant relationship with SY, with greatest effect direct and positive (0.549) and lower indirect effect of $\mathrm{P}(-0.225)$.

Under the $150 \mathrm{~kg} \mathrm{~N} \mathrm{ha}^{-1}$ the greatest correlation was observed via $\mathrm{S}(0.92)$. $\mathrm{S}$ is defined in the early crop stages and since no residual effect from the previous crop was possible (given that they were removed from the area), it is suggested that the reason for this correlation is the direct effect of this trait $(0.665)$ and the indirect effect via $\mathrm{P}$ (0.237). P had positive and significant correlation with SY (0.807) via indirect effect of S (0.531). PW showed lower correlation (0.455) via indirect and direct effect of $S(0.206)$.

Thus, the two major traits contributing both directly and indirectly to IAC Milenio's SY are P and S regardless of the nitrogen level. Therefore breeders can use either direct or indirect selection for these traits to enhance SY in this cultivar.

During year II (2017), for IPR Tuiuiú under zero nitrogen, significant positive correlations with SY were observed for several variables: H, P, PW, and SW. Reason for this effect is attributed to the previous planted crop (rye) residual effect via crop residues (Table 6). $\mathrm{H}$ and PW was correlated with SY mainly via P.

For P and SW the main correlation cause with SY was the direct effect of the trait, supporting that when the positive correlation exists along with a direct positive effect the target trait will be impacted. This fact also supports direct selection for these traits (Vale et al., 2009). For SW, the direct effect (0.412) was greater than the overall correlation (0.205), which can be explained by the indirect negative effect of SP $(-0.148)$.

Under the 50, 100 and 150 doses, P, SP, PW and SW showed positive and significant relation with SY. Apart for the PW influence, all these were direct influence of the trait. At $150 \mathrm{~kg} \mathrm{~N}^{-1}, \mathrm{P}$ and SP effects were more pronounced in the overall correlation.

SP presents high heritability coefficient, thus environmental effects on the trait expression are diminished (Vale et al., 2009). For both cultivars can be noted that this trait was key to SY determination, not being largely affected by the environment ( $\mathrm{N}$ dosages).

H showed significant and positive relation with SY under the zero, 50 and 150-N treatment levels, but the main effect was indirect via $\mathrm{P}\left(0\right.$ and $\left.50 \mathrm{~kg} \mathrm{~N} \mathrm{ha}^{-1}\right)$ and $\mathrm{PW}\left(150 \mathrm{~kg} \mathrm{~N} \mathrm{ha}^{-1}\right)$.

PW showed positive correlation with SY under all nitrogen doses, with the main effect being indirect via P under 0 , $50,100 \mathrm{~kg} \mathrm{~N} \mathrm{ha}^{-1}$. At $150 \mathrm{~kg} \mathrm{~N} \mathrm{ha}^{-1}$, the direct effect of the trait was more important.

Similar correlation patterns were observed in both years (I and II), but their magnitude varied. In 2016, the main yield component affecting IAC Milenio's SY was S, via direct and indirect effect of another traits, depending on the nitrogen level. P is an important trait for SY when nitrogen is applied, given that it has a direct effect on this trait. Under the zero-nitrogen treatment it showed negative correlation with SY, via indirect effect of S though

In 2017, a greater number of correlations with SY was observed for IPR Tuiuiú. P was the main yield component under all nitrogen doses and influenced directly $\left(0,50\right.$ and $\left.100 \mathrm{~kg} \mathrm{~N} \mathrm{ha}^{-1}\right)$ and indirectly $\left(150 \mathrm{~kg} \mathrm{~N}^{-1}\right) \mathrm{SY}$. These findings support similar results published by Farinelli and Lemos (2010), who suggested breeding schemes based on this trait.

It is noted that in a crop-livestock system, regardless of the nitrogen fertilization level (from zero up to $150 \mathrm{~kg} N$ $\mathrm{ha}^{-1}$ ), the traits that affect SY most are the same (Kurek et al., 2001; Cabral et al., 2011; Lad, Longmei, \& Borle, 2017), when cultivars are analyzed separately. As a consequence, it can be concluded that the main yield components of dry beans are not impacted by environmental changes (i.e. variance for these traits are higher than the environment variance), information that should guide and accelerate future breeding efforts for this species. 
Table 6. Pearson correlation coefficient estimates and associated indirect and direct effects of plant height $(\mathrm{H})$, first pod height (FPH), pods per plant (P), seed per pod (SP), seeds per plant (S), pod weight per plant (PW), and thousand seeds weight (SW) on seed yield per plant (SY) for dry beans, IAC Milenio cultivar (Year 2, $\mathrm{n}=96$ plants) under four nitrogen levels $\left(0,50,100\right.$ and $\left.150 \mathrm{~kg} \mathrm{ha}^{-1}\right)$. Abelardo Luz-SC, 2017

\begin{tabular}{|c|c|c|c|c|c|c|}
\hline \multicolumn{7}{|l|}{ Year II } \\
\hline \multirow{2}{*}{ Effect } & \multicolumn{6}{|c|}{ Yield Components } \\
\hline & $\mathrm{H}$ & FPH & $\mathrm{P}$ & SP & PW & SW \\
\hline \multicolumn{7}{|l|}{ Dosis 0} \\
\hline DIRECT ON SY & -0.029 & 0.006 & 0.997 & 0.409 & -0.046 & 0.412 \\
\hline INDIRECT VIA H & - & 0.001 & -0.008 & -0.003 & -0.007 & 0.002 \\
\hline INDIRECT VIA FPH & 0.000 & - & -0.001 & 0.000 & -0.001 & 0.000 \\
\hline INDIRECT VIA P & 0.277 & -0.122 & - & -0.153 & 0.648 & -0.064 \\
\hline INDIRECT VIA SP & 0.045 & -0.028 & -0.063 & - & -0.064 & -0.148 \\
\hline INDIRECT VIA PW & -0.011 & 0.009 & -0.030 & 0.007 & - & 0.002 \\
\hline INDIRECT VIA SW & -0.033 & 0.029 & -0.026 & -0.149 & -0.015 & - \\
\hline r & $0.250^{*}$ & $-0.105^{\mathrm{ns}}$ & $0.869^{* *}$ & $0.112^{\mathrm{ns}}$ & $0.515^{* *}$ & $0.205^{*}$ \\
\hline $\mathrm{R}^{2}$ & 0.977 & & & & & \\
\hline \multicolumn{7}{|l|}{ Dosis 50} \\
\hline DIRECT ON SY & -0.009 & 0.021 & 0.811 & 0.224 & 0.083 & 0.291 \\
\hline INDIRECT VIA H & - & -0.002 & -0.004 & -0.002 & -0.003 & 0.001 \\
\hline INDIRECT VIA FPH & 0.004 & - & 0.000 & 0.002 & 0.000 & -0.003 \\
\hline INDIRECT VIA P & 0.332 & -0.016 & - & 0.185 & 0.524 & 0.050 \\
\hline INDIRECT VIA SP & 0.053 & 0.024 & 0.051 & - & 0.019 & -0.051 \\
\hline INDIRECT VIA PW & 0.029 & -0.002 & 0.054 & 0.007 & - & 0.022 \\
\hline INDIRECT VIA SW & -0.033 & -0.040 & 0.018 & -0.066 & 0.079 & - \\
\hline $\mathrm{r}$ & $0.375^{* *}$ & $-0.015^{\mathrm{ns}}$ & $0.929^{* *}$ & $0.350^{* *}$ & $0.701^{* *}$ & $0.311^{* *}$ \\
\hline $\mathrm{R}^{2}$ & 0.977 & & & & & \\
\hline \multicolumn{7}{|l|}{ Dosis 100} \\
\hline DIRECT ON SY & -0.025 & 0.026 & 0.793 & 0.212 & 0.161 & 0.283 \\
\hline INDIRECT VIA H & - & -0.004 & -0.005 & -0.001 & -0.005 & 0.001 \\
\hline INDIRECT VIA FPH & 0.004 & - & -0.006 & 0.004 & -0.005 & 0.001 \\
\hline INDIRECT VIA P & 0.150 & -0.179 & - & 0.006 & 0.675 & -0.042 \\
\hline INDIRECT VIA SP & 0.010 & 0.032 & 0.002 & - & 0.008 & -0.015 \\
\hline INDIRECT VIA PW & 0.030 & -0.030 & 0.137 & 0.006 & - & 0.030 \\
\hline INDIRECT VIA SW & -0.007 & 0.010 & -0.015 & -0.020 & 0.053 & - \\
\hline $\mathrm{r}$ & $0.162^{\mathrm{ns}}$ & $-0.145^{\mathrm{ns}}$ & $0.907^{* *}$ & $0.207^{* *}$ & $0.888^{* *}$ & $0.258^{*}$ \\
\hline $\mathrm{R}^{2}$ & 0.971 & & & & & \\
\hline \multicolumn{7}{|l|}{ Dosis 150} \\
\hline DIRECT ON SY & 0.014 & -0.033 & 0.224 & 0.202 & 0.677 & 0.219 \\
\hline INDIRECT VIA H & - & 0.002 & 0.002 & 0.005 & 0.004 & 0.001 \\
\hline INDIRECT VIA FPH & -0.004 & - & 0.001 & -0.003 & 0.000 & -0.003 \\
\hline INDIRECT VIA P & 0.026 & -0.004 & - & 0.049 & 0.097 & -0.068 \\
\hline INDIRECT VIA SP & 0.070 & 0.018 & 0.045 & - & 0.062 & -0.034 \\
\hline INDIRECT VIA PW & 0.199 & -0.006 & 0.295 & 0.207 & - & 0.162 \\
\hline INDIRECT VIA SW & 0.023 & 0.017 & -0.066 & -0.037 & 0.052 & - \\
\hline $\mathrm{r}$ & $0.328^{* *}$ & $-0.007^{\mathrm{ns}}$ & $0.499^{* *}$ & $0.424^{* *}$ & $0.893^{* *}$ & $0.277^{* *}$ \\
\hline $\mathrm{R}^{2}$ & 0.867 & & & & & \\
\hline
\end{tabular}

\section{Conclusion}

Yield components correlation profiles are different for the analyzed cultivars.

For the cultivar IAC Milenio, the trait that impacted most grain yield was number of grain per plant, via indirect and direct effect of other traits. The effect is independent of nitrogen levels.

For the cultivar IPR Tuiuiú, number of pods per plant was the trait that most impacted final yield, for all nitrogen fertilization schemes. 
The traits pod weight and thousand seeds weight were significant for yield increase only for the IPR Tuiuiú cultivar.

\section{References}

Adami, P. F., Pelissari, A., Moraes, A. D., Modolo, A. J., Assmann, T. S., Franchin, M. F., \& Cassol, L. C. (2012). Grazing intensities and poultry litter fertilization levels on corn and black oat yield. Pesquisa Agropecuária Brasileira, 47(3), 360-368. https://doi.org/10.1590/S0100-204X2012000300007

Afonso, R. J., Arf, O., Costa, D. S. D., Barbosa, R. M., Buzetti, S., Sá, M. E. D., \& Rodrigues, R. A. F. (2011). Combinações de fontes de nitrogênio no desenvolvimento e rendimento do feijoeiro. Pesquisa Agropecuária Tropical, 391-398. https://doi.org/10.5216/pat.v41i3.12360

Arf, M. V., Buzetti, S., Arf, O., Kappes, C., Ferreira, J. P., Gitti, D. D. C., \& Yamamoto, C. J. T. (2011). Fontes e épocas de aplicação de nitrogênio em feijoeiro de inverno sob sistema plantio direto. Pesquisa Agropecuária Tropical, 430-438. https://doi.org/10.5216/pat.v41i3.9706

Cabral, P. D. S., Soares, T. C. B., Lima, A. B. P., Soares, Y. J. B., \& de Silva, J. A. (2011). Análise de trilha do rendimento de grãos de feijoeiro (Phaseolus vulgaris L.) e seus componentes. Revista Ciência Agronômica, 42(1), 132-138. https://doi.org/10.1590/S1806-66902011000100017

Carvalho, C. G. P., Arias, C. A. A., de Toledo, J. F. F., de Oliveira, M. F., \& Vello, N. A. (2002). Correlações e análise de trilha em linhagens de soja semeadas em diferentes épocas. Pesquisa Agropecuária Brasileira, 37(3), 311-320. https://doi.org/10.1590/S0100-204X2002000300012

Carvalho, F. D., Lorencetti, C., \& Benin, G. (2004). Estimativas e implicações da correlação no melhoramento vegetal (p. 142). Pelotas: UFPel.

Coimbra, J. L. M., Benin, G., Vieira, E. A., Oliveira A. C. F., Carvalho, F. I. F., Guidolin, A. F., \& Soares, A. P. (2005). Conseqüências da multicolinearidade sobre a análise de trilha em canola. Ciência Rural, 35(2), 347-352. https://doi.org/10.1590/S0103-84782005000200015

CONAB (Companhia Nacional de Abastecimento). (2018). Acompanhamento da safra brasileira de grãos. Safra 2017/18-Sétimo levantamento.

Costa, J. C. G., Zimmermann, M. J. O., \& Melhoramento, G. (1988). In M. J. O. Zimmermann, M. Rocha, \& T. Yamada (Eds.), A cultura do feijoeiro: Fatores que afetam a produtividade.

Cruz, C. D. (2013). Genes: A software package for analysis in experimental statistics and quantitative genetics. Acta Scientiarum. Agronomy, 35(3), 271-276. https://doi.org/10.4025/actasciagron.v35i3.21251

Cruz, C. D., \& Carneiro, P. C. S. (2012). Modelos biométricos aplicados ao melhoramento genético. Universidade Federal de Viçosa, Brazil.

Demari, G. H., de Souza, V. Q., Carvalho, I. R., Nardino, M. \& Follmann, D. N. (2015). Feijão em época não preferencial submetido a doses de nitrogênio e seu impacto nos caracteres agronômicos. Enciclopédia Biosfera, 11(21), 1102.

Duarte, T. S., Peil, R. M., \& Montezano, E. M. (2008). Crescimento de frutos do meloeiro sob diferentes relações fonte: dreno. Hortic. bras, 26(3). https://doi.org/10.1590/S0102-05362008000300010

Farinelli, R., \& Lemos, L. B.. (2010). Características agronômicas de genótipos de feijoeiro cultivados nas épocas da seca e das águas. Bragantia, 69(2), 361-366. https://doi.org/10.1590/S0006-87052010000200013

Freitas, T. G. G. D., Silva, P. S. L. E., Dovale, J. C., \& Silva, E. (2016). Green Bean Yield and Path Analysis in Cowpea Landraces. Revista Caatinga, 29(4), 866-877. https://doi.org/10.1590/1983-21252016v29n411rc

Gomes, F. G., de Sá, M. E., \& Valério Filho, W. V. (2008). Nitrogênio no feijoeiro em sistema de plantio direto sobre gramíneas. Acta Scientiarum: Agronomy, 387-395.

Gondim, T. C. O., Rocha, V. S., Sediyama, C. S., \& Miranda, G. V. (2008). Análise de trilha para componentes do rendimento e caracteres agronômicos de trigo sob desfolha. Pesquisa Agropecuária Brasileira, 43(4), 487-493. https://doi.org/10.1590/S0100-204X2008000400007

Hoogerheide, E. S. S., Vencovsky, R., Farias, F. J. C., Freire, E. C., \& Arantes, E. M. (2007). Correlações e análise de trilha de caracteres tecnológicos e a produtividade de fibra de algodão. Pesquisa Agropecuária Brasileira, 42(10), 1401-1405. https://doi.org/10.1590/S0100-204X2007001000005

Kappes, C., Wruck, F., Carvalho, M. A. C., \& Yamashita, O. (2008). Feijão comum: características morfo-agronômicas de cultivares. Embrapa Arroz e Feijão-Artigo em anais de congresso (ALICE). 
Congresso nacional de pesquisa de Feijão, 9, 2008, Campinas. Ciência e tecnologia na cadeia produtiva do feijão. Campinas: Instituto Agronômico.

Kurek, A. J., Carvalho, F. D., Assmann, I. C., Marchioro, V. S., \& Cruz, P. J. (2001). Análise de trilha como critério de seleção indireta para rendimento de grãos em feijão. Revista Brasileira de Agrociência, 7(1), 29-32.

Lad, D. B., Longmei, N., \& Borle, U. M. (2017). Studies on Genetic Variability, Association of Characters and Path Analysis in French Bean (Phaseolus vulgaris L.). Int. J. Pure App. Biosci., 5(6), 1065-1069. https://doi.org/10.18782/2320-7051.5757

Lopes, S. J., Storck, L., Perin Damo, H., Brum, B., \& Dos Santos, V. J. (2007). Relações de causa e efeito em espigas de milho relacionadas aos tipos de híbridos. Ciência Rural, 37(6). https://doi.org/10.1590/S0103 $-84782007000600005$

Montgomery, D. C., \& Peck, E. A. (1981). Introduction to linear regression analysis (p. 504). New York: J. Wiley.

Moreira, G. B., Pegoraro, R. F., Vieira, N., Borges, I., \& Kondo, M. K. (2013). Desempenho agronômico do feijoeiro com doses de nitrogênio em semeadura e cobertura. Revista Brasileira de Engenharia Agricola e Ambiental-Agriambi, 17(8). https://doi.org/10.1590/S1415-43662013000800003

Moura, M. M., Carneiro, P. C. S., de Souza Carneiro, J. E., \& Cruz, C. D. (2013). Potencial de caracteres na avaliação da arquitetura de plantas de feijão de feijão. Pesquisa Agropecuária Brasileira, 48(4), 417-425. https://doi.org/10.1590/S0100-204X2013000400010

Nogueira, A. P. O., Sediyama, T., de Sousa, L. B., Hamawaki, O. T., Cruz, C. D., Pereira, D. G., \& Matsuo, É. (2012). Análise de trilha e correlações entre caracteres em soja cultivada em duas épocas de semeadura. Bioscience Journal, 28(6).

Ramalho, M. A. P., Santos, J. D., \& Zimmermann, M. D. O. (1993). Genética quantitativa em plantas autógamas: aplicações ao melhoramento do feijoeiro. Goiânia: UFG.

SEAB (Secretária de Estado da Agricultura e do Abastecimento do Paraná). (2017). Feijão-Análise da Conjuntura Agropecuária.

Silva, T. R. B. D., Lemos, L. B., \& Crusciol, C. A. C. (2011). Produtividade e características tecnológicas de cultivares de feijão em resposta à calagem superficial em plantio direto. Bragantia, 196-205. https://doi.org/10.1590/S0006-87052011000100026

Souza, A. B. D., Andrade, M. J. B. D., Vieira, N. M. B., \& Albuquerque, A. D. (2008). Densidades de semeadura e níveis de NPK e calagem na produção do feijoeiro sob plantio convencional, em Ponta Grossa, Paraná. Pesquisa Agropecuária Tropical, 38(1), 39-43.

Teixeira, I. R., Andrade, M. D., Carvalho, J. D., Morais, A. D., \& Corrêa, J. B. D. (2000). Resposta do feijoeiro (Phaseolus vulgaris L. cv. Pérola) a diferentes densidades de semeadura e doses de nitrogênio. Ciência e Agrotecnologia, 24(2), 399-408.

Vale, N. M., Barili, L. D., da Rocha, F., Rozzetto, D. S., Pereira, T. P., Coimbra, J. L. M., ... Bertoldo, J. G. (2009). Métodos de semeadura empregados como critério de avaliação fenotípica em melhoramento de feijão. Biotemas, 22(4), 73-81. https://doi.org/10.5007/2175-7925.2009v22n4p73

Viana, T. O., Vieira, N. M. B., Moreira, G. B. L., Batista, R. O., Carvalho, S. J. P., \& Rodrigues, H. F. F. (2011). Adubação do feijoeiro cultivado no norte de Minas Gerais com nitrogênio e fósforo. Revista Ceres, $58(1)$. https://doi.org/10.1590/S0034-737X2011000100017

Zilio, M., Coelho, C. M. M., Souza, C. A., Santos, J. C. P., \& Miquelluti, D. J. (2011). Contribuição dos componentes de rendimento na produtividade de genótipos crioulos de feijão (Phaseolus vulgaris L.). Revista Ciência Agronômica, 42(2), 429-438. https://doi.org/10.1590/S1806-66902011000200024

\section{Copyrights}

Copyright for this article is retained by the author(s), with first publication rights granted to the journal.

This is an open-access article distributed under the terms and conditions of the Creative Commons Attribution license (http://creativecommons.org/licenses/by/4.0/). 